\title{
Influence of water injection atomization on the performance of water-lubricated single screw air compressor
}

\author{
Yanan $\mathrm{Li}^{1 *}$, Jingfu Wang ${ }^{1}$, Yuting $\mathrm{Wu}^{1}$ and Biao Lei ${ }^{1}$ \\ ${ }^{1}$ Faculty of Environment and Life, Beijing University of Technology, Beijing, China
}

\begin{abstract}
As the whole society attaches great importance to the safety of food and medicine, and hydrogen fuel cell and new energy vehicle industry rises suddenly, people's requirements for clean compressed air is more and more urgent. Water-lubricated single screw compressor is an ideal choice for providing clean air because of the advantages of balanced force, a long service life, and high efficiency, and the influencing factors of its performance have received more and more attention. This study established a mathematical model of the working process of a water-lubricated single-screw air compressor to study the relationship between its performance and the water injection atomization. The model considered leakage and heat transfer between water and moist air and analysed the influences of droplet diameter and rotation speed on compressor performance. The results showed that water injection atomization can effectively reduce the discharge temperature, make the compression process of the compressor tend to isothermal compression. Under rated conditions, a reduction in the droplet diameter from $0.25 \mathrm{~mm}$ to $0.15 \mathrm{~mm}$ reduced the discharge temperature of the compressor, improved the volume efficiency, and improved the adiabatic efficiency by $31 \mathrm{~K}, 2.6 \%$, and $8.2 \%$, respectively.
\end{abstract}

\section{Introduction}

There have in recent years been increasingly urgent requirements for improved quality of compressed air, and in particular, oil-free clean air. The water-lubricated single screw air compressor relies on water injection to replace oil injection and plays the role of cooling, sealing, lubrication and noise reduction for the compressed air, so that the compressor can operate in an isothermal compression process and effectively reduce the energy consumption of the machine [1]. At the same time, the radial and axial air force borne by the screw of the single screw compressor can be automatically balanced, and there is no triangle leakage area problem. Therefore, water lubricated single screw air compressor is an efficient means to produce pure oil-free compressed air.

The research on the water lubricated compressor is mainly focused on the twin screw compressor [2-4], and there have been few studies on the performance of water-lubricated single-screw air compressors. Li et al., in a study of the influence of different meshing pair lines on the hydrodynamic lubrication parameters of a singlescrew compressor through theoretical and experimental methods, found that the optimal water-lubrication effect was achieved by adopting the multiple cylindrical meshing pair [5][6]. However, so far, the effect of water injection atomization on compressor performance has not been studied. Yang et al., researched the relationship between water injection flow rate and the performance of single-screw water vapour compressor, showed that atomization of water injection could improve the performance of the vapour compressor [7]. Although the mechanical structures of vapour compressors and air compressors are similar, they have quite different working conditions and thermal performance.

Therefore, the focus of the current study was on the water-lubricated single-screw air compressor. Water injection atomization is an important parameter affecting water lubricated single screw air compressor. Therefore, the present study established a thermodynamic model to represent the working process of the water-lubricated single-screw air compressor to examine the influences of the diameter of the atomized droplets injected into the compressor and the rotation speed on the performance of the compressor. The results of the present study can provide theoretical guidance for the design and optimization of a water-lubricated single-screw air compressor.

\section{Mathematical model of water- lubricated single screw air compressor}

Water lubricated single screw air compressor is mainly composed of screw rotor, gate rotors and shell. The ratio of tooth number of screw rotor and gate rotor is $11 / 6$. The screw rotates under the drive of the motor, and the volume of the working chamber changes with the change of the angle of screw. As shown in Fig. 1, the working process can be divided into four stages: suction, sealing, compression and discharge.

\footnotetext{
* Corresponding author: lyn2018@emails.bjut.edu.cn
} 


\subsection{Assumptions}

The actual working process of water-lubricated single screw air compressor is very complicated. The working medium involves three kinds of fluids, namely liquid water, water vapour and dry air. In order to simplify the calculation, the following assumptions were made when establishing the mathematical model of the working process:

(1) The changes of potential energy and kinetic energy of fluids were neglected.

(2) There were uniform distributions of temperature and pressure in each fluid in the control volume, and the state parameters of the working medium changed periodically with changes in the angle of the screw rotation.

(3) Water vapour was always in a saturated state.

(5) The pressure pulsation in the suction process was neglected.

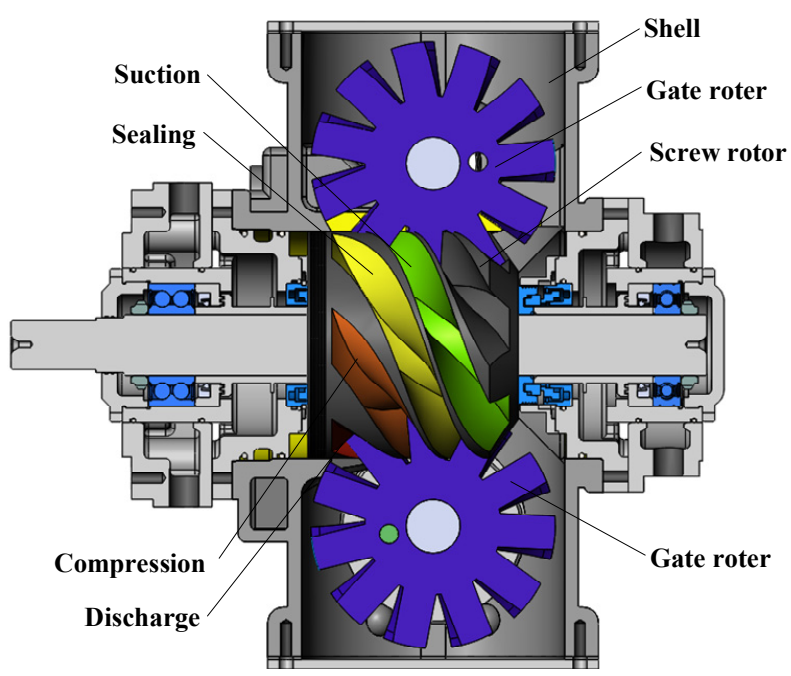

Fig. 1. Schematic diagram of working process of waterlubricated single screw air compressor

\subsection{Governing equations}

One of the working screw grooves was selected as the control volume which is shown in Fig. 2. The nomenclature in this article is shown in Fig.3.

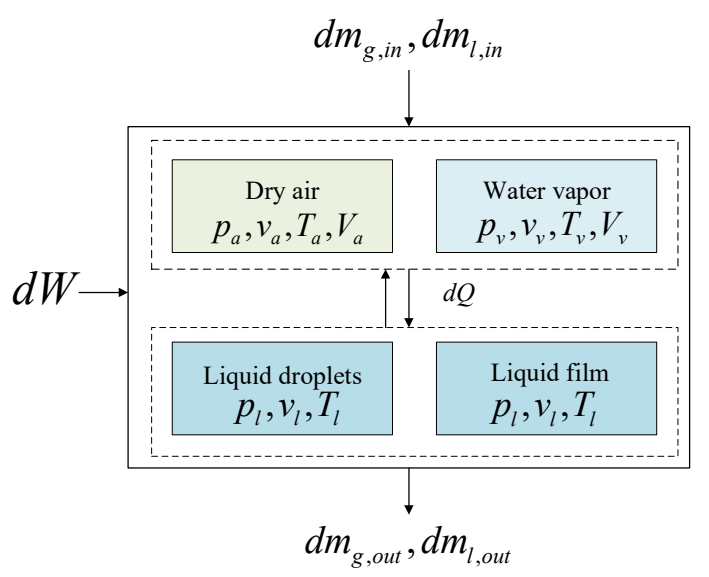

Fig. 2. The control volume

\begin{tabular}{|c|c|}
\hline \multicolumn{2}{|c|}{ Nomenclature } \\
\hline$C$ & flow coefficient \\
\hline$c$ & specific heat, $\mathrm{J} /(\mathrm{kg} \cdot \mathrm{K})$ \\
\hline$d$ & Diameter, mm \\
\hline$f$ & slip factor \\
\hline$h$ & specific enthalpy, $\mathrm{J} / \mathrm{kg}$ \\
\hline$k$ & $\begin{array}{l}\text { heat convection coefficient, } \\
\mathrm{W} /\left(\mathrm{m}^{2} \cdot \mathrm{K}\right)\end{array}$ \\
\hline$m$ & mass of fluid, $\mathrm{kg}$ \\
\hline in & mass flow rate of fluid, $\mathrm{kg} / \mathrm{s}$ \\
\hline$N u$ & Nusselt number \\
\hline$n$ & rotating speed of screw rotor, rpm \\
\hline$P$ & power, W \\
\hline $\operatorname{Pr}$ & Prandlt number \\
\hline$p$ & pressure, $\mathrm{Pa}$ \\
\hline$Q$ & heat transfer quantity, $\mathrm{J}$ \\
\hline $\operatorname{Re}$ & Reynolds number \\
\hline$S$ & area, $\mathrm{m}^{2}$ \\
\hline$T$ & temperature, $\mathrm{K}$ \\
\hline$q_{\mathrm{v}}$ & volume flow of fluid, $\mathrm{m}^{3} / \mathrm{s}$ \\
\hline$u$ & specific internal energy, $\mathrm{J} / \mathrm{kg}$ \\
\hline$V$ & volume, $\mathrm{m}^{3}$ \\
\hline \multirow[t]{2}{*}{$v$} & velocity, $\mathrm{m} / \mathrm{s}$ \\
\hline & specific volume, $\mathrm{m}^{3} / \mathrm{kg}$ \\
\hline$W$ & Work, J \\
\hline \multicolumn{2}{|c|}{ Greek symbols } \\
\hline$\alpha$ & void fraction \\
\hline$\xi$ & atomization rate \\
\hline$\theta$ & rotating angle of screw rotor, rad \\
\hline$\lambda$ & thermal conductivity, $\mathrm{W} /(\mathrm{m} \cdot \mathrm{K})$ \\
\hline$\chi$ & mass fraction of gas \\
\hline$\rho$ & density, $\mathrm{kg} / \mathrm{m}^{3}$ \\
\hline \multicolumn{2}{|c|}{ Subscripts } \\
\hline$a$ & dry air \\
\hline ad & adiabatic compression \\
\hline$d$ & discharge \\
\hline$e$ & evaporation \\
\hline$g$ & gas mixture \\
\hline in & inflow of control volume \\
\hline$l$ & liquid water \\
\hline out & outflow of control volume \\
\hline real & real value \\
\hline th & theoretical value \\
\hline$v$ & saturated water vapor \\
\hline
\end{tabular}

Fig. 3. The nomenclature

According to the conservation of mass and energy, the differential equation of mass and internal energy of each component with the change of screw rotation angle can be given. Where, the mass conservation equation of moist air is: 


$$
\frac{d m_{g}}{d \theta}=\frac{d m_{g, \text { in }}}{d \theta}-\frac{d m_{g, \text { out }}}{d \theta}
$$

The mass conservation equation of liquid water is:

$$
\frac{d m_{l}}{d \theta}=\frac{d m_{l, \text { in }}}{d \theta}-\frac{d m_{l, o u t}}{d \theta}
$$

The mass conservation equation of water vapour is:

$$
\frac{d m_{v}}{d \theta}=\frac{d m_{v, \text { in }}}{d \theta}-\frac{d m_{v, \text { out }}}{d \theta}+\frac{d m_{e}}{d \theta}
$$

The energy conservation equation of dry air is:

$$
\frac{d\left(m_{a} u_{a}\right)}{\mathrm{d} \theta}=\sum \frac{d m_{a, \text { in }}}{\mathrm{d} \theta} h_{a, \text { in }}-\sum \frac{d m_{a, \text { out }}}{\mathrm{d} \theta} h_{a, \text { out }}-\frac{d Q_{a}}{\mathrm{~d} \theta}-p_{a} \frac{d V_{a}}{d \theta}
$$

The energy conservation equation of liquid water is:

$$
\frac{d\left(m_{l} u_{l}\right)}{\mathrm{d} \theta}=\sum \frac{d m_{l, \text { in }}}{\mathrm{d} \theta} h_{l, \text { in }}-\sum \frac{d m_{l, \text { out }}}{\mathrm{d} \theta} h_{l, \text { out }}-\frac{d Q_{l}}{\mathrm{~d} \theta}
$$

\section{3 leakage model}

As shown in Fig. 4, the single-screw air compressor has nine leakage paths.

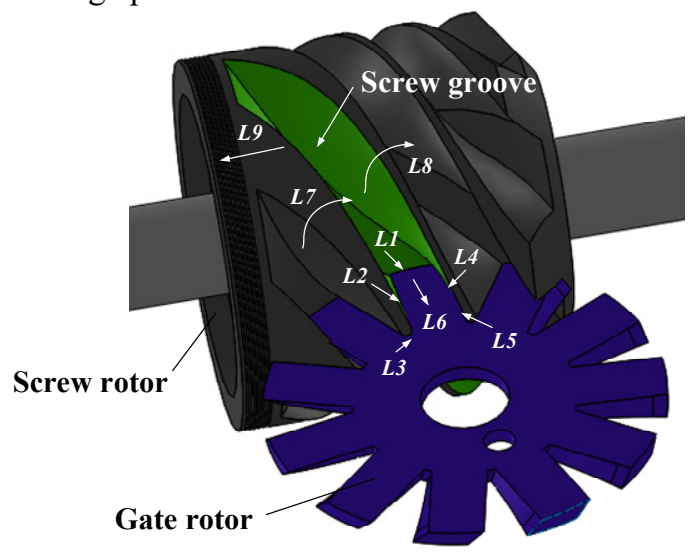

Fig. 4. Leakage paths of a single-screw compressor

In this paper, the fluid passing through each leakage path is considered as two-phase laminar flow, and it was assumed that the mass fraction of the gas in the leakage channel is equal to the mass fraction of the gas in the compression chamber. The leakage equation can be expressed as follows [8]:

$$
\left\{\begin{array}{l}
\frac{d m_{g}}{d \theta}=C_{l e a} \alpha S \rho_{g} v_{g} / \omega \\
\frac{d m_{l}}{d \theta}=C_{l e a}(1-\alpha) S \rho_{l} v_{l} / \omega \\
v_{g}=\sqrt{2\left(h_{l}-h_{2}\right)} \\
v_{l}=v_{g} / f \\
f=0.4+0.6 \sqrt{\frac{\rho_{l}}{\rho_{g}}+0.4\left(\frac{1}{\chi}-1\right)} \sqrt{1+0.4\left(\frac{1}{\chi}-1\right)} \\
\alpha=\frac{1}{1+f\left(\frac{1}{\chi}-1\right) \frac{\rho_{g}}{\rho_{l}}}
\end{array}\right.
$$

\subsection{Heat transfer model}

In this paper, it is assumed that the water injected into the compressor exists in two forms: droplets and film, the liquid film uniformly attached to the wall surface of the control volume. Therefore, the total heat transfer between water and moist air is the sum of convective heat transfer between moist air and droplets as well as liquid film:

$$
\frac{d Q}{d \theta}=\frac{d Q_{\text {fim }}}{d \theta}+\frac{d Q_{\text {drop }}}{d \theta}
$$

The forced convection heat transfer formula can be used to calculate the heat transfer between moist air and water, and the heat transfer coefficient can be determined by the $N u$. Where, the change of heat transfer between moist air and liquid film with the angle of screw rotation can be calculated by the following equation:

$$
\begin{gathered}
d Q_{\text {film }} / d \theta=k_{\text {film }} S_{\text {film }}\left(T_{g}-T_{l}\right) / \omega \\
k_{\text {film }}=N u_{\text {film }} \lambda_{g} / d_{e} \\
N u_{\text {film }}=0.023 R e_{\text {film }}^{0.8} P r_{\text {film }}^{0.3} c_{r}
\end{gathered}
$$

The change of heat transfer between the moist air and the droplet with the angle of screw rotation can be calculated by the following equation:

$$
\begin{gathered}
d Q_{\text {drop }} / d \theta=k_{\text {drop }} S_{\text {drop }}\left(T_{g}-T_{l}\right) / \omega \\
k_{\text {drop }}=N u_{\text {drop }} \lambda_{g} / d_{\text {drop }} \\
N u_{\text {drop }}=2+0.6 R e_{\text {drop }}^{0.5} \operatorname{Pr}_{\text {drop }}^{0.33}
\end{gathered}
$$

In order to simplify the calculation, the atomization rate $\zeta$ is introduced, which is equal to the percentage of droplet mass in the total mass of liquid water. Its value range is between 0 and 1 .

\subsection{Performance parameters}

Volume efficiency is an important evaluation index of compressor performance, which reflects the perfect utilization degree of compressor geometry size and evaluates the impact of leakage on compressor performance. Its calculation formula is as follows:

$$
\eta_{v}=q_{v, \text { real }} / q_{v, \text { th }}
$$

The theoretical volume flow rate is:

$$
q_{v, t h}=2 n z_{1} V_{i n}
$$

The adiabatic efficiency of the compressor is the ratio between the power required for isentropic adiabatic compression and the actual power consumption of the compressor:

$$
\eta_{a d}=P_{a d} / P_{s}
$$

The calculation equation of isentropic adiabatic compression power of the compressor is:

$$
P_{a d}=\dot{m}_{\text {real }}\left(h_{d, a d}-h_{i n}\right)
$$

The power consumed by compressed moist air is:

$$
P_{s, g}=\dot{m}_{\text {real }} \int_{p_{i}}^{p_{d}}-v d p
$$

The power consumed by compressed liquid water is:

$$
P_{s, l}=\dot{m}_{l}\left(p_{d}-p_{\text {in }}\right) / \rho_{l}
$$

Then, the actual power consumption of the compressor is:

$$
P_{s}=P_{s, g}+P_{s, l}
$$

The specific power of the compressor is the power consumed by the gas flow per unit volume, that is, the 
actual power consumption ratio to the actual volume flow rate:

$$
P_{s p}=P_{s} / q_{v, \text { real }}
$$

\subsection{Calculation parameters}

Table 1. Main structure and operating parameters of the waterlubricated single screw air compressor

\begin{tabular}{cc}
\hline Parameters & Value \\
\hline Screw rotor diameter & $147 \mathrm{~mm}$ \\
Gate rotor diameter & $158 \mathrm{~mm}$ \\
Centre distance & $117.6 \mathrm{~mm}$ \\
Rotation speed & $3000 \mathrm{rpm}$ \\
Rated power & $11 \mathrm{KW}$ \\
Rated discharge pressure & $0.467 \mathrm{MPa}$ \\
\hline
\end{tabular}

\section{Results and discussions}

\subsection{Influence of different droplet diameters on the discharge temperature}

Under a constant water injection flow rate, the heat transfer area could be effectively increased by reducing the diameter of the atomized droplet, resulting in an increase in heat transfer. As shown in Fig. 5, a decrease in the droplet diameter from $0.25 \mathrm{~mm}$ to $0.15 \mathrm{~mm}$ resulted in a decrease in discharge temperature of $31 \mathrm{~K}$ under the rated working condition and a water injection flow rate of $70 \mathrm{~L} / \mathrm{min}$. Meanwhile, the discharge temperature of water lubricated single screw air compressor is obviously lower than that of oil lubricated compressor.

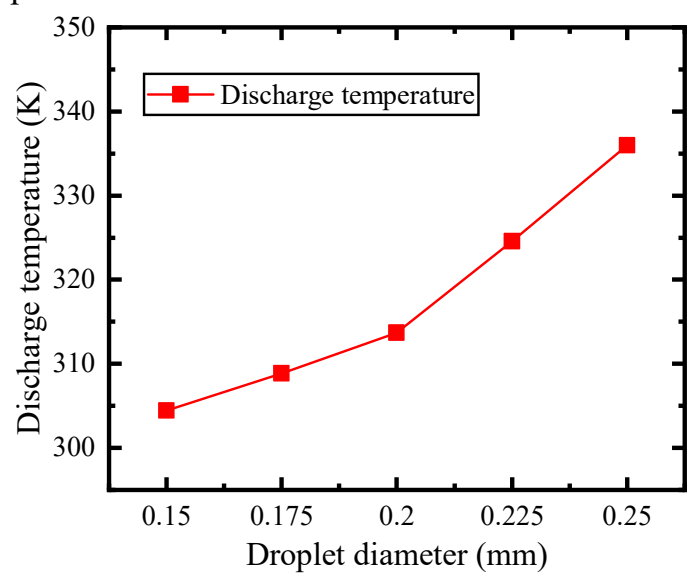

Fig.5. Influence of different droplet diameters on the discharge temperature of a water-lubricated single screw air compressor

\subsection{Influence of different droplet diameters on the $p$ - $V$ diagram}

As shown in Fig. 6, under a droplet diameter of $0.25 \mathrm{~mm}$, the shape of the compression process curve approximated that of adiabatic compression. The shape of the $\mathrm{p}-\mathrm{V}$ curve gradually moved away from that of adiabatic compression to that of isothermal compression with decreasing droplet diameter.

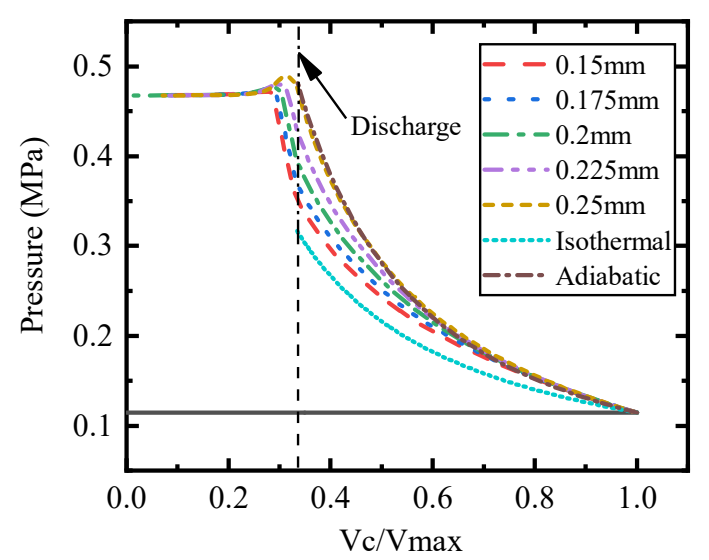

Fig.6. Influence of different droplet diameters of a waterlubricated single screw air compressor on the $p$ - $V$ diagram

\subsection{Influence of different droplet diameters on volume efficiency and water -gas ratio}

As shown in Fig. 7(a), under a constant droplet diameter, there was an increase in the volume efficiency with increasing rotation speed. At a droplet diameter of 0.2 $\mathrm{mm}$, the volume efficiency increased from $67.1 \%$ to $79.7 \%$ with an increase in rotation speed from $1,500 \mathrm{rpm}$ to $3,000 \mathrm{rpm}$, although the rate of increase gradually slowed. This result can be attributed to the volume efficiency being related to the amount of leakage. On the one hand, the leakage rate for a single working screw groove was mostly constant at different rotation speeds, with leakage time and leakage quantity inversely related to rotation speed. On the other hand, as shown in Fig. 7(b), there was a decrease in the water-gas ratio in the screw groove as the rotation speed increased, which affected the sealing and heat transfer and increased leakage. Therefore, the increase in the rate of volume efficiency declined with increasing rotation speed.

Under a constant rotation speed, since a small droplet diameter increased heat transfer and slowed the rise in pressure in the screw groove, there was a reduction in the leakage rate and an increase in volume efficiency. The highest volume efficiency of $80.8 \%$ was achieved under a droplet diameter of $0.15 \mathrm{~mm}$ and rotation speed of $3,000 \mathrm{rpm}$. The effect of the reduction in droplet diameter on volume efficiency becomes more obvious with increasing rotation speed. A decrease in the droplet diameter from $0.25 \mathrm{~mm}$ to $0.15 \mathrm{~mm}$ resulted in increases in volume efficiency of $0.8 \%$ and $2.6 \%$ at rotation speeds of 1,500 rpm and 3,000 rpm, respectively.

Under a constant water injection flow rate, the watergas ratio was only related to the rotation speed and was independent of the droplet diameter. As shown in Fig. 7 (b), the water-gas ratio decreased from $5.9 \%$ to $2.5 \%$ with an increase in rotation speed from $1,500 \mathrm{rpm}$ to $3,000 \mathrm{rpm}$, exceeding that of an oil-lubricated air compressor. 


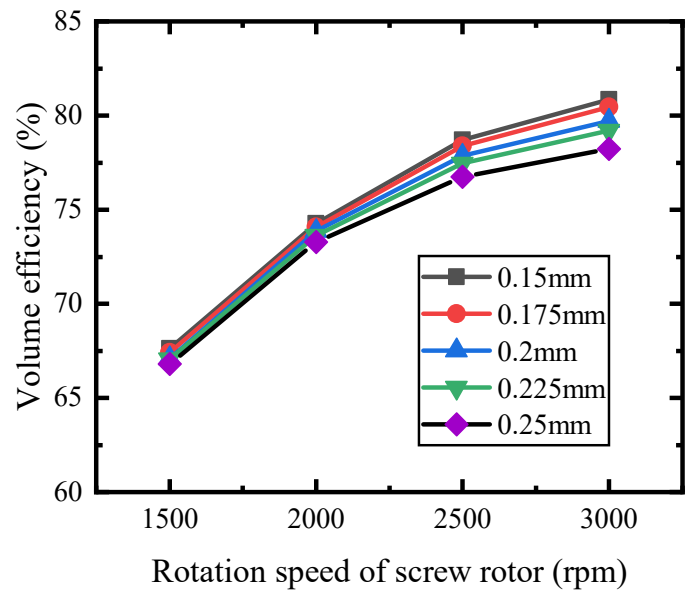

(a) Volume efficiency

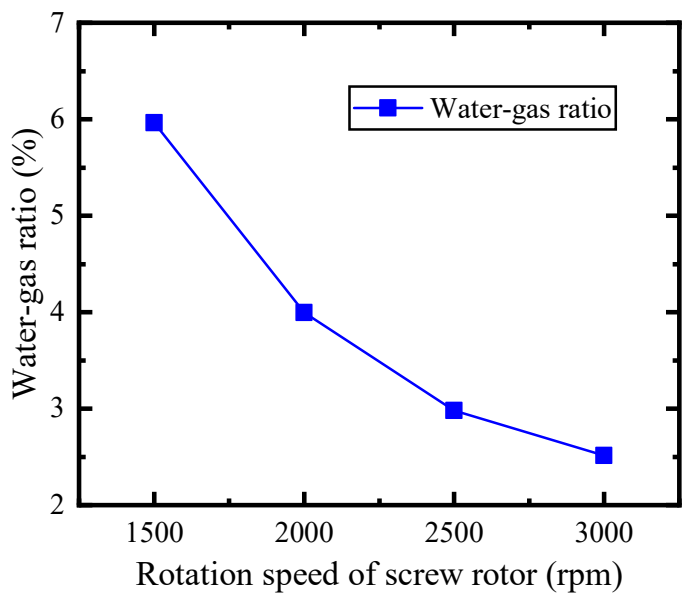

(b) Water-gas ratio

Fig.7. Influence of rotation speed and droplet diameter on volume efficiency and water -gas ratio of a water-lubricated single screw air compressor

\subsection{Influence of different droplet diameters on adiabatic efficiency}

Fig. 8 shows the influence of rotation speed and droplet diameter on adiabatic efficiency. As shown in Fig. 8, under a droplet diameter of $0.25 \mathrm{~mm}$, there was first an increase in adiabatic efficiency followed by a decrease. This result could be attributed to an increase in volume efficiency with increases in rotation speed. Meanwhile, power consumption increased as the water-air ratio decreased. Above a certain threshold of rotation speed, the effect of the increase in power consumption exceeded that of the increase in volume efficiency, resulting in a decline in adiabatic efficiency. When the droplet diameter is constant, it can be inferred that adiabatic efficiency increased with increasing rotation speed to a maximum, following which it decreased.

Under a constant rotation speed, adiabatic efficiency increased with decreasing droplet diameter. The maximum adiabatic efficiency of $77.4 \%$ was achieved at a droplet diameter of $0.15 \mathrm{~mm}$ and rotation speed of $3,000 \mathrm{rpm}$. Under a high rotation speed, the influence of the droplet diameter on the adiabatic efficiency is more obvious than that under a low speed. A reduction in the droplet diameter from $0.25 \mathrm{~mm}$ to $0.15 \mathrm{~mm}$ resulted in increase in adiabatic efficiency of $2.25 \%$ and $8.17 \%$ under rotation speeds of $1,500 \mathrm{rpm}$ and $3,000 \mathrm{rpm}$, respectively.

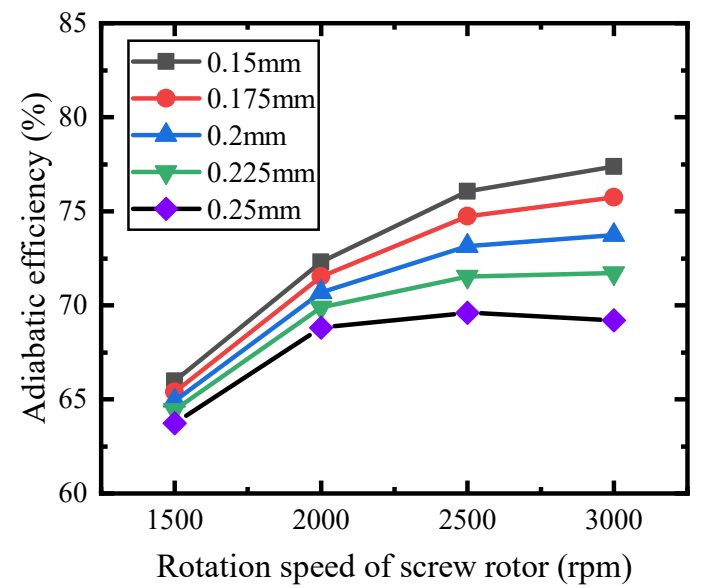

Fig.8. Influence of rotation speed and droplet diameter on adiabatic efficiency of a water-lubricated single screw air compressor

\subsection{Influence of different droplet diameters on specific power}

Fig. 9 shows the influence of rotation speed and droplet diameter on specific power. Under a droplet diameter of $0.25 \mathrm{~mm}$, the specific power first decreased and then increased with increasing rotation speed. Under the remaining droplet diameters investigated, the specific power decreased monotonously with increasing rotation speed. This result could be attributed to the gradual slowing in the increase in volume efficiency with increasing rotation speed, and the power consumption due to leakage resulting from the reduction in the watergas ratio increased. Under a condition of the increase in volume efficiency equaling the increase of the power consumption, specific power tended to remain constant. However, specific power increased when the latter exceeded the former.

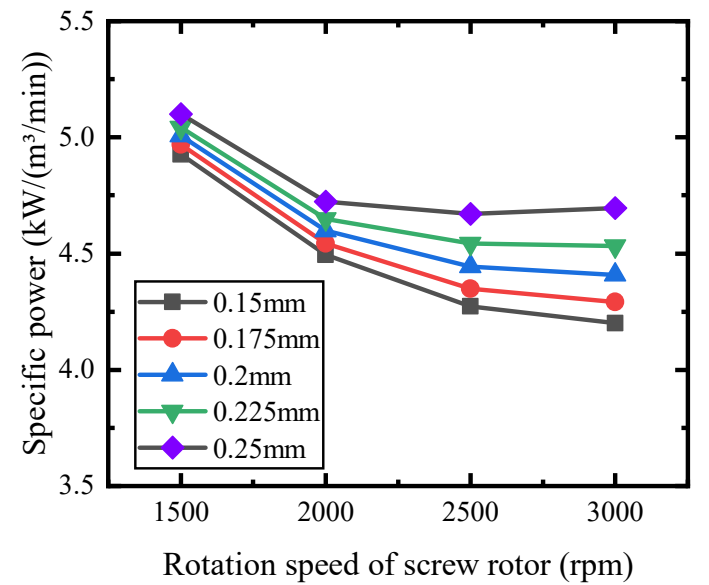

Fig.9. Influence of rotation speed and droplet diameter on specific power of a water-lubricated single screw air compressor

Under a constant rotation speed, specific power decreased with decreasing droplet diameter. Under a high rotation speed, the specific power can be more reduced by reducing droplet diameter than that under a 
low rotation speed. A decrease in droplet diameter from $0.25 \mathrm{~mm}$ to $0.15 \mathrm{~mm}$ resulted in decreases in specific power by $0.17 \mathrm{kw} /(\mathrm{m} 3 / \mathrm{min})$ and $0.49 \mathrm{~kW} /(\mathrm{m} 3 / \mathrm{min})$ at $1,500 \mathrm{rpm}$ and $3,000 \mathrm{rpm}$, respectively. This result indicated that the influence of heat exchange on power consumption and leakage become increasingly obvious with increasing rotation speed.

\section{Conclusions}

The present study constructed a mathematical model of the working process of a water-lubricated single screw air compressor to identify the influences of diameter of liquid droplets and rotation speed on the performance of the compressor. The present study came to the following conclusions:

(1) Water injection atomization can effectively reduce the discharge temperature, make the compression process of the compressor tend to isothermal compression, and improve the performance of the compressor. Under rated working conditions, the volume efficiency and specific power of the compressor reached $80.8 \%$ and $4.2 \mathrm{~kW} /(\mathrm{m} 3 / \mathrm{min})$, respectively under a water injection flow rate of $70 \mathrm{~L} / \mathrm{min}$ and a reduction in the droplet diameter to $0.15 \mathrm{~mm}$.

(2) The smaller the droplet diameter after atomization is, the better the heat transfer effect is, and the better the performance of the compressor can be improved.

(3) The increase of rotation speed is beneficial to improve the efficiency of the compressor. However, due to the decrease of water-air ratio, the cooling and sealing effect becomes worse. Therefore, the effect of increasing the speed on the efficiency of the compressor is gradually weakened.

\section{Acknowledgments}

The authors are grateful for the financial support provided by the National Key R\&D Program of China (NO. 2016YFC0700403), Beijing Municipal Natural Science Foundation (NO. 3181001).

\section{References}

1. 1. W. Shen, J. Tong. Engineering Thermodynamics. (Higher Education Press, Beijing, 2007)

2. J. Li, H. Wu, B. Wang, Z. Xing, P. Shu, Research on the performance of water-injection twin screw compressor, Applied Thermal Engineering, 29(2009): 3401-3408.

3. C. Wang, Z. Xing, W. Chen, Q. Yang, Z. He, Development of an oil free water-lubricated twinscrew air compressor, Applied Thermal Engineering,143(2018): 396-402.

4. C. Wang, Z. Xing, W. Chen, S. Sun, Z. He, Analysis of the leakage in a water-lubricated twin-screw air compressor, Applied Thermal Engineering, 155(2019): 217-225.
5. T. Li, R. Huang, Q. Feng, W. Wu, F. Liu, X. Yu, Hydrodynamic lubricating characteristics of water flooded single screw compressors based on two types of meshing pair profile, Proceedings of the Institution of Mechanical Engineers, Part J: Journal of Engineering Tribology, 230(9) (2016): 1092-1106.

6. T. Li, Z. Liu, R. Huang, F. Liu, Q. Feng, X. Yu, Research of the hydrodynamic lubrication characteristics of different meshing pair profiles in water-flooded single screw compressors, Proceedings of the Institution of Mechanical Engineers, Part A: Journal of Power and Energy, 230(3) (2016): 247-259.

7. J. Yang, C. Zhang, Z. Zhang, L. Yang, W. Lin, Study on mechanical vapor recompression system with wet compression single screw compressor, Applied Thermal Engineering, 103(2016): 205-211.

8. L. Shen, W. Wang, Y. Wu, B. Lei, R. Zhi, Y. Lu, J. Wang, C. Ma, A study of clearance height on the performance of single screw expanders in smallscale organic Rankine cycles, Energy, 153(2018): 45-55. 